\title{
Deodhar Elements in Kazhdan-Lusztig Theory
}

\author{
Brant Jones $^{\dagger}$ \\ Department of Mathematics, One Shields Avenue, University of California, Davis, CA 95616
}

\begin{abstract}
The Kazhdan-Lusztig polynomials for finite Weyl groups arise in representation theory as well as the geometry of Schubert varieties. It was proved very soon after their introduction that they have nonnegative integer coefficients, but no simple all positive interpretation for them is known in general. Deodhar has given a framework, which generally involves recursion, to express the Kazhdan-Lusztig polynomials in a very attractive form.

We use a new kind of pattern-avoidance that can be defined for general Coxeter groups to characterize when Deodhar's algorithm yields a non-recursive combinatorial formula for Kazhdan-Lusztig polynomials $P_{x, w}(q)$ of finite Weyl groups. This generalizes results of Billey-Warrington which identified the 321-hexagon-avoiding permutations, and Fan-Green which identified the fully-tight Coxeter groups. We also show that the leading coefficient known as $\mu(x, w)$ for these Kazhdan-Lusztig polynomials is always either 0 or 1 . Finally, we generalize the simple combinatorial formula for the Kazhdan-Lusztig polynomials of the 321-hexagon-avoiding permutations to the case when $w$ is hexagon avoiding and maximally clustered.

Résumé. Les polynômes de Kazhdan-Lusztig $P_{x, w}(q)$ des groupes de Weyl finis apparaissent en théorie des représentations, ainsi qu'en géométrie des variétés de Schubert. Il a été démontré peu après leur introduction qu'ils avaient des coefficients entiers positifs, mais on ne connaît toujours pas d'interprétation combinatoire simple de cette propriété dans le cas général. Deodhar a proposé un cadre donnant un algorithme, en général récursif, calculant des formules attractives pour les polynômes de Kazhdan-Lusztig. Billey-Warrington ont démontré que cet algorithme est non récursif lorsque $w$ évite les hexagones et les 321 et qu'il donne des formules combinatoires simples.

Nous introduisons une notion d'évitement de schémas dans les groupes de Coxeter quelconques nous permettant de généraliser les résultats de Billey-Warrington à tout groupe de Weyl fini. Nous montrons que le coefficient de tête $\mu(x, w)$ de ces polynômes de Kazhdan-Lusztig est toujours 0 ou 1 . Cela généralise aussi des résultats de Fan-Green qui identifient les groupes de Coxeter complètement serrés. Enfin, en type A, nous obtenons une classe plus large de permutations évitant la récursion.
\end{abstract}

Keywords: Kazhdan-Lusztig polynomial, pattern avoidance, 321-hexagon, freely-braided, maximally-clustered, 2sided weak Bruhat order

\footnotetext{
${ }^{\dagger}$ The author received support from NSF grants DMS-9983797 and DMS-0636297. 


\section{Introduction}

The Kazhdan-Lusztig polynomials introduced in (KL79) have interpretations for finite Weyl groups as Poincaré polynomials for intersection cohomology of Schubert varieties (KL80) and as a $q$-analogue of the multiplicities for Verma modules (BB81; BK81). From these interpretations, it is known that the Kazhdan-Lusztig polynomials have nonnegative integer coefficients. However, their combinatorial structure remains obscure and no simple all positive formula for the coefficients is known in general.

Deodhar (Deo90) has proposed a framework for determining the Kazhdan-Lusztig polynomials which can be described for an arbitrary Coxeter group. The framework gives the Kazhdan-Lusztig polynomials in the form of a combinatorial generating function, but generally involves summation over a certain recursively defined set. The algorithm he describes is shown to work for all Coxeter groups where the Kazhdan-Lusztig polynomials are known to have nonnegative integer coefficients which includes Weyl groups and the Coxeter groups associated to crystallographic Kac-Moody groups.

In this paper, we define embedded factor pattern avoidance for Coxeter groups and use it to characterize the elements for which Deodhar's algorithm yields a simple combinatorial formula for the KazhdanLusztig polynomials of finite Weyl groups. We call such elements Deodhar.

We show that the leading coefficient of the Kazhdan-Lusztig polynomial $P_{x, w}(q)$ known as $\mu(x, w)$ is always either 0 or 1 when $w$ is a Deodhar element. This coefficient is used in Kazhdan and Lusztig's construction of cells for Weyl group and Hecke algebra representations.

We also provide a non-recursive description for the bounded admissible sets of masks used by Deodhar's framework to calculate the Kazhdan-Lusztig polynomials $P_{x, w}(q)$ of type $A$, in the case when $w$ is hexagon avoiding and maximally clustered. The maximally clustered elements were introduced in (Los07), and include the fully-commutative and freely-braided elements as a subset. The maximallyclustered hexagon-avoiding permutations are characterized by avoiding the seven classical permutation patterns $\{3421,4312,4321,46718235,46781235,56718234,56781234\}$.

For more details as well as proofs of the results given in this paper, please see (BJ07), (Jon07a) and (Jon07b).

\section{Heaps and Deodhar's theorem}

Let $W$ be a Coxeter group with generating set $S$ and relations of the form $\left(s_{i} s_{j}\right)^{m(i, j)}=1$. The Coxeter graph for $W$ is the graph on the generating set $S$ with edges connecting $s_{i}$ and $s_{j}$ labeled $m(i, j)$ for all pairs $i, j$ with $m(i, j)>2$. Note that if $m(i, j)=3$ it is customary to leave the corresponding edge unlabeled.

We view the symmetric group $S_{n}$ as a Coxeter group of type $A$ with generators $S=\left\{s_{1}, \ldots, s_{n-1}\right\}$ and relations of the form $\left(s_{i} s_{i \pm 1}\right)^{3}=1$ together with $\left(s_{i} s_{j}\right)^{2}=1$ for $|i-j| \geq 2$. The Coxeter graph of type $A_{n-1}$ has the form

$$
\bullet_{1}-\bullet_{2}-\bullet_{3}-\ldots-\bullet_{n-1} .
$$

We may also refer to elements in the symmetric group by the 1 -line notation $w=\left[w_{1} w_{2} \ldots w_{n}\right]$ where $w$ is the bijection mapping $i$ to $w_{i}$. Then the generators $s_{i}$ are the adjacent transpositions interchanging the entries $i$ and $i+1$ in the 1-line notation. If $w=\left[w_{1} \ldots w_{n}\right]$, and $p=\left[p_{1} \ldots p_{k}\right]$ is another permutation in $S_{k}$ for $k \leq n$, then we say $w$ contains the permutation pattern $p$ whenever there exists a subsequence $1 \leq i_{1}<i_{2}<\ldots<i_{k} \leq n$ such that

$$
w_{i_{a}}<w_{i_{b}} \text { if and only if } p_{a}<p_{b}
$$


for all $1 \leq a<b \leq k$. We call $\left(i_{1}, i_{2}, \ldots, i_{k}\right)$ the pattern instance. For example, [ $\left.\underline{5} 32 \underline{41}\right]$ contains the pattern [321] in several ways, including the underlined subsequence. If $w$ does not contain the pattern $p$, we say that $w$ avoids $p$. There are 1-line notations for Coxeter types $B$ and $D$ that carry a notion of pattern containment generalizing this classical definition.

An expression is any product of generators from $S$ and the length $l(w)$ is the minimum length of any expression for the element $w$. Such a minimum length expression is called reduced. Each element $w \in W$ can have several different reduced expressions representing it and we denote the set of all reduced expressions for $w$ by $R(w)$. For example, we have $s_{2} s_{3} s_{1} s_{2} \in R([3412])$. Given $w \in W$, we represent reduced expressions for $w$ in sans serif font, say $\mathrm{w}=\mathrm{w}_{1} \mathrm{w}_{2} \cdots \mathrm{w}_{p}$ where each $\mathrm{w}_{i} \in S$. We call any expression of the form $s_{i} s_{i \pm 1} s_{i}$ a short-braid after A. Zelevinski (see (Fan98)). This name reflects the fact that we are not considering any longer braid even if $m(i, j)>3$, but we caution the reader that some authors have used the term short-braid to refer to a commutation move between two entries $s_{i}$ and $s_{j}$ where $m(i, j)=2$. We say that $x<w$ in Bruhat order if a reduced expression for $x$ appears as a subword that is not necessarily consecutive, of a reduced expression for $w$. If $s_{i}$ appears as the last factor in any reduced expression for $w$, then we say that $s_{i}$ is a descent for $w$; otherwise, $s_{i}$ is an ascent for $w$.

It is a theorem of Tits (Tit69) that every reduced expression for an element $w$ of a Coxeter group can be obtained from any other by applying a sequence of braid moves of the form

$$
\underbrace{s_{i} s_{j} s_{i} s_{j} \cdots}_{m(i, j)} \mapsto \underbrace{s_{j} s_{i} s_{j} s_{i} \cdots}_{m(i, j)}
$$

where $s_{i}$ and $s_{j}$ are generators in $S$ that appear in the reduced expression for $w$, and each factor in the move has $m(i, j)$ letters. Let the support of an element $w \in W$, denoted $\operatorname{supp}(w)$, be the set of all generators appearing in any reduced expression for $w$, which is well-defined by Tits' theorem. We say that the element $w$ is connected if $\operatorname{supp}(w)$ is connected in the Coxeter graph of $W$.

We define an equivalence relation on the set of reduced expressions for an element by saying that two reduced expressions are in the same commutativity class if one can be obtained from the other by a sequence of commuting moves of the form $s_{i} s_{j} \mapsto s_{j} s_{i}$ where $m(i, j)=2$. If the reduced expressions for an element $w$ form a single commutativity class, then we say $w$ is fully-commutative.

Several interesting properties of Schubert varieties, Kazhdan-Lusztig polynomials, and Bruhat order can be characterized by pattern avoidance. For example, the property of a permutation being short-braid avoiding in type $A$ is characterized by avoiding the pattern [321], as first noted by (BJS93). Hence, the fully commutative elements in $S_{n}$ are enumerated by the Catalan numbers (SS85). Also, checking a permutation of length $n$ for a subsequence of length 3 can be done in $O\left(n^{3}\right)$ time whereas looking at all reduced words for a typical permutation takes an exponential amount of time in $n$.

\subsection{Heaps}

Just as Young tableaux have facilitated many combinatorial arguments by adding an additional dimension to the 1-line notation of a permutation, heaps allow us to visualize reduced expressions as a set of lattice points in two dimensions, while maintaining the pertinent information about relations among the generators.

If $w=w_{1} \cdots w_{k}$ is a reduced expression, then following (Ste96) we define a partial ordering on the indices $\{1, \cdots, k\}$ by the transitive closure of the relation $i \lessdot j$ if $i<j$ and $\mathbf{w}_{i}$ does not commute with $\mathrm{w}_{j}$. We label each element $i$ of the poset by the corresponding generator $\mathrm{w}_{i}$. It follows quickly 
from the definition that if $\mathrm{w}$ and $\mathrm{w}^{\prime}$ are two reduced expressions for a permutation $w$ that are in the same commutativity class then the labeled posets of $w$ and $w^{\prime}$ are isomorphic. This isomorphism class of labeled posets is called the heap of $\mathrm{w}$, where $\mathrm{w}$ is a reduced expression representative for a commutativity class of $w$. In particular, if $w$ is fully-commutative then it has a single commutativity class, and so there is a unique heap of $w$.

As in (BW01), we will represent a heap of a permutation as a set of lattice points embedded in $\mathbb{N}^{2}$. To do so, we assign coordinates $(x, y) \in \mathbb{N}^{2}$ to each entry of the labeled Hasse diagram for the heap of $w$ in such a way that:

(1) An entry represented by $(x, y)$ is labeled by the Coxeter generator $s_{i}$ in the heap if and only if $x=i$, and

(2) If an entry represented by $(x, y)$ is greater than an entry represented by $\left(x^{\prime}, y^{\prime}\right)$ in the heap then $y>y^{\prime}$.

Since the Coxeter graph of type $A$ is a path, it follows from the definition that $(x, y)$ covers $\left(x^{\prime}, y^{\prime}\right)$ in the heap if and only if $x=x^{\prime} \pm 1, y>y^{\prime}$, and there are no entries $\left(x^{\prime \prime}, y^{\prime \prime}\right)$ such that $x^{\prime \prime} \in\left\{x, x^{\prime}\right\}$ and $y^{\prime}<y^{\prime \prime}<y$. Hence, we can completely reconstruct the edges of the Hasse diagram and the corresponding heap poset from a lattice point representation. This representation enables us to make arguments "by picture" that would otherwise be difficult to formulate.

Example 2.1 One lattice point representation of the heap of $w=s_{2} s_{3} s_{1} s_{2} s_{4}$ is shown below, together with the labeled Hasse diagram for the unique heap poset of $w$.
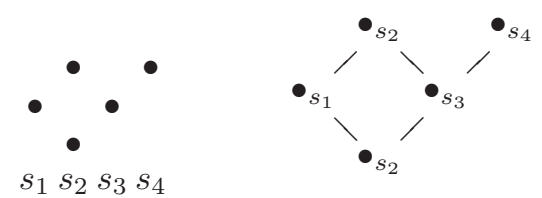

We now describe a notion of containment for Coxeter group elements. We say that

$$
f:\left\{s_{1}, \ldots, s_{k}\right\} \rightarrow\left\{s_{1}, \ldots, s_{n}\right\}
$$

is a Coxeter embedding if it is an injective map of Coxeter generators such that for each $m \geq 2$, we have

$$
\left(s_{i} s_{j}\right)^{m}=1 \text { if and only if }\left(f\left(s_{i}\right) f\left(s_{j}\right)\right)^{m}=1 .
$$

We can view this as a map of group elements by extending it to a word homomorphism which is then applied to any reduced expression in the Coxeter group generated by $\left\{s_{1}, \ldots, s_{k}\right\}$.

Suppose $W$ is a Coxeter group, and $w, y \in W$. Let $W_{y}$ be the parabolic subgroup whose generators are determined by the support of $y$. If there exists a Coxeter embedding $f: W_{y} \rightarrow W$ such that $w$ contains $f(y)$ as a factor, then we say that $w$ contains $y$ as an embedded factor.

To illustrate, $w=s_{2} s_{3} s_{1} s_{2} s_{4}$ from Example 2.1 contains $s_{1} s_{2} s_{3}$ as an embedded factor under the Coxeter embedding that sends $s_{i} \mapsto s_{i+1}$, but $w$ does not contain $s_{1} s_{2} s_{1}$ as an embedded factor. 


\subsection{Deodhar's theorem}

Given any Coxeter group $W$, we can form the Hecke algebra $\mathcal{H}$ over the ring $\mathbb{Z}\left[q^{1 / 2}, q^{-1 / 2}\right]$ with basis $\left\{T_{w}: w \in W\right\}$, and relations:

$$
\begin{aligned}
T_{s} T_{w} & =T_{s w} \text { for } l(s w)>l(w) \\
\left(T_{s}\right)^{2} & =(q-1) T_{s}+q T_{1}
\end{aligned}
$$

where $T_{1}$ corresponds to the identity element. In particular, this implies that

$$
T_{w}=T_{\mathrm{w}_{1}} T_{\mathrm{w}_{2}} \ldots T_{\mathrm{w}_{p}}
$$

whenever $\mathrm{w}_{1} \mathrm{w}_{2} \ldots \mathrm{w}_{p}$ is a reduced expression for $w$. Also, it follows from (2.2) that the basis elements $T_{w}$ are invertible. Observe that when $q=1$, the Hecke algebra $\mathcal{H}$ becomes the group algebra of $W$.

Kazhdan and Lusztig (KL79) described another basis for $\mathcal{H}$ that is invariant under the ring involution on the Hecke algebra defined by $\bar{q}=q^{-1}, \overline{T_{w}}=\left(T_{w^{-1}}\right)^{-1}$, where we denote the involution with an overline. This basis, denoted $\left\{C_{w}^{\prime}: w \in W\right\}$, has important applications in representation theory and algebraic geometry (KL80). The Kazhdan-Lusztig polynomials $P_{x, w}(q)$ describe how to change between these bases of $\mathcal{H}$ :

$$
C_{w}^{\prime}=q^{-\frac{1}{2} l(w)} \sum_{x \leq w} P_{x, w}(q) T_{x} .
$$

The $C_{w}^{\prime}$ are defined uniquely to be the Hecke algebra elements that are invariant under the involution and have expansion coefficients as above, where $P_{x, w}$ is a polynomial in $q$ with

$$
\text { degree } P_{x, w}(q) \leq \frac{l(w)-l(x)-1}{2}
$$

for all $x<w$ in Bruhat order and $P_{w, w}(q)=1$. We use the notation $C_{w}^{\prime}$ to be consistent with the literature because there is already a related basis denoted $C_{w}$.

The following open conjecture motivates Deodhar's theorem. This conjecture is known to be true in a number of special cases including when the Coxeter group is finite or affine.

Conjecture 2.2 (KL79) The coefficients of $P_{x, w}(q)$ are nonnegative in the Hecke algebra associated to any Coxeter group.

Deodhar (Deo90) studied the case when $C_{w}^{\prime}$ can be written simply as a product of $C_{s_{i}}^{\prime}$ 's. In this case, he also gives nice combinatorial formulas for all the polynomials $P_{x, w}(q)$. We will describe Deodhar's defect statistic and his theorem in terms of masks on reduced expressions.

Fix a reduced expression $\mathrm{w}=\mathrm{w}_{1} \mathrm{w}_{2} \cdots \mathrm{w}_{k}$. Define a mask $\sigma$ associated to the reduced expression $\mathrm{w}$ to be any binary vector $\left(\sigma_{1}, \cdots, \sigma_{k}\right)$ of length $k=l(w)$. Every mask corresponds with a subexpression of $\mathrm{w}$ defined by $\mathrm{w}^{\sigma}=\mathrm{w}_{1}^{\sigma_{1}} \cdots \mathrm{w}_{k}^{\sigma_{k}}$ where

$$
\mathrm{w}_{j}^{\sigma_{j}}= \begin{cases}\mathrm{w}_{j} & \text { if } \sigma_{j}=1 \\ \text { id } & \text { if } \sigma_{j}=0 .\end{cases}
$$

Each $\mathrm{w}^{\sigma}$ is a product of generators so it determines an element of $W$. For $1 \leq j \leq k$, we also consider initial sequences of masks denoted $\sigma[j]=\left(\sigma_{1}, \cdots, \sigma_{j}\right)$, and the corresponding initial subexpressions 
$\mathrm{w}^{\sigma[j]}=\mathrm{w}_{1}^{\sigma_{1}} \cdots \mathrm{w}_{j}^{\sigma_{j}}$. In particular, we have $\mathrm{w}^{\sigma[k]}=\mathrm{w}^{\sigma}$. The mask $\sigma$ is proper if it does not consist of all 1 entries, since $\mathrm{w}^{(1, \ldots, 1)}=\mathrm{w}$ which is the fixed reduced expression for $w$.

We say that a position $j$ (for $2 \leq j \leq k$ ) of the fixed reduced expression $w$ is a defect with respect to the mask $\sigma$ if

$$
l\left(\mathrm{w}^{\sigma[j-1]} \mathrm{w}_{j}\right)<l\left(\mathrm{w}^{\sigma[j-1]}\right) .
$$

Note that the defect status of position $j$ does not depend on the value of $\sigma_{j}$. Let $d_{w}(\sigma)$ denote the number of defects of $\mathrm{w}$ for a mask $\sigma$. We will use the notation $d(\sigma)=d_{\mathrm{w}}(\sigma)$ if the reduced word $\mathrm{w}$ is fixed.

Deodhar's framework gives a combinatorial interpretation for the Kazhdan-Lusztig polynomial $P_{x, w}(q)$ as the generating function for masks $\sigma$ on a reduced expression w with respect to the defect statistic $d(\sigma)$. We begin by considering subsets of the set

$$
\mathcal{S}=\{0,1\}^{l(w)} .
$$

of all possible masks on w. For $\mathcal{E} \subset \mathcal{S}$, we define a prototype for $P_{x, w}(q)$ :

$$
P_{x}(\mathcal{E})=\sum_{\substack{\sigma \in \mathcal{E} \\ \mathrm{w}^{\sigma}=x}} q^{d(\sigma)}
$$

and a corresponding prototype for the Kazhdan-Lusztig basis element $C_{w}^{\prime}$ :

$$
h(\mathcal{E})=q^{-\frac{1}{2} l(w)} \sum_{\sigma \in \mathcal{E}} q^{d(\sigma)} T_{\mathrm{w}^{\sigma}} .
$$

Definition 2.3 (De090) Fix $\mathrm{w}=\mathrm{w}_{1} \mathrm{w}_{2} \ldots \mathrm{w}_{k}$. We say that $\mathcal{E} \subset \mathcal{S}$ is admissible on $\mathrm{w}$ if:

1. $\mathcal{E}$ contains $\sigma=(1,1, \ldots, 1)$.

2. $\mathcal{E}=\tilde{\mathcal{E}}$ where $\tilde{\sigma}=\left(\sigma_{1}, \sigma_{2}, \ldots, \sigma_{k-1}, 1-\sigma_{k}\right)$.

3. $h(\mathcal{E})=\overline{h(\mathcal{E})}$ is invariant under the involution on the Hecke algebra.

We say that $\mathcal{E}$ is bounded on $w$ if $P_{x}(\mathcal{E})$ has degree $\leq \frac{1}{2}(l(w)-l(x)-1)$ for all $x<w$ in Bruhat order.

Theorem 2.4 (De090) Let $x, w$ be elements in any Coxeter group $W$, and fix a reduced expression $\mathrm{w}$ for $w$. If $\mathcal{E} \subset \mathcal{S}$ is bounded and admissible on $\mathrm{w}$, then

$$
P_{x, w}(q)=P_{x}(\mathcal{E})=\sum_{\substack{\sigma \in \mathcal{E} \\ \mathbf{w}^{\sigma}=x}} q^{d(\sigma)}
$$

and hence

$$
C_{w}^{\prime}=h(\mathcal{E})=q^{-\frac{1}{2} l(w)} \sum_{\sigma \in \mathcal{E}} q^{d(\sigma)} T_{\mathbf{w}^{\sigma}} .
$$

We say that an element $w$ is Deodhar if $\mathcal{S}$ is bounded on any (equivalently, every) reduced expression w of $w$. Deodhar has shown that the set $\mathcal{S}$ is always admissible so Theorem 2.4 gives an all positive combinatorial formula without recursion for the Kazhdan-Lusztig polynomials associated to $w$. 
Example 2.5 Assume $s_{2} s_{1} s_{3} s_{2}$ is a reduced expression in a Coxeter group. The reduced expression/mask pair

$$
\mathrm{w}=\left[\begin{array}{cccc}
s_{2} & s_{1} & s_{3} & s_{2} \\
1 & 0 & 0 & 0
\end{array}\right]
$$

has a defect in position 4. One can verify that the set $\mathcal{S}$ of all $2^{4}$ masks on $\mathrm{w}=s_{2} s_{1} s_{3} s_{2}$ is bounded, so w is Deodhar.

Several other characterizations of the Deodhar elements are given in the following result.

\begin{tabular}{|c|c|c|}
\hline Type & Coxeter graph & Embedded factor patterns \\
\hline $\begin{array}{l}I_{2}(m) \\
m \geq 3\end{array}$ & $\bullet \bullet_{1} \frac{m}{\bullet} \bullet_{2}$ & $s_{1} s_{2} s_{1}, s_{2} s_{1} s_{2}$ (short braids) \\
\hline$A_{7}$ & $\bullet \bullet_{1}-\bullet_{2}-\bullet_{3}-\bullet_{4}-\bullet_{5}-\bullet_{6}-\bullet_{7}$ & $s_{5} s_{6} s_{7} s_{3} s_{4} s_{5} s_{6} s_{2} s_{3} s_{4} s_{5} s_{1} s_{2} s_{3}$ (hexagon) \\
\hline$B_{7} / C_{7}$ & $\bullet$ & $s_{4} s_{5} s_{6} s_{2} s_{3} s_{4} s_{5} s_{1} s_{2} s_{3} s_{4} s_{0} s_{1} s_{2}$ ( $B$-hexagon) \\
\hline$D_{6}$ & 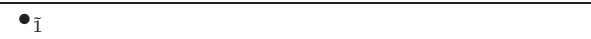 & $s_{3} s_{4} s_{5} s_{1} s_{2} s_{3} s_{4} s_{1} s_{2} s_{3} s_{1}\left(H E X_{4}\right)$ \\
\hline$D_{7}$ & $\bullet^{\bullet} \bullet_{1}$ & $\begin{array}{l}s_{3} s_{4} s_{5} s_{6} s_{2} s_{3} s_{4} s_{5} s_{1} s_{2} s_{3} s_{4} s_{1} s_{2} s_{3}\left(H E X_{2}\right) \\
s_{4} s_{5} s_{6} s_{2} s_{3} s_{4} s_{5} s_{1} s_{2} s_{3} s_{4} s_{1} s_{2} s_{1}\left(H E X_{3 a}\right) \\
s_{1} s_{4} s_{5} s_{6} s_{2} s_{3} s_{4} s_{5} s_{1} s_{2} s_{3} s_{4} s_{1} s_{2}\left(H E X_{3 b}\right)\end{array}$ \\
\hline$D_{8}$ & & $\begin{array}{l}s_{4} s_{5} s_{6} s_{7} s_{3} s_{4} s_{5} s_{6} s_{2} s_{3} s_{4} s_{5} s_{1} s_{1} s_{2} s_{3} s_{4} \\
\text { (diamond, to be avoided as a 1-line pattern) }\end{array}$ \\
\hline$E_{6}$ & $\bullet \bullet_{0}-\bullet_{1}-\bullet_{2}-\bullet_{3}-\bullet_{4}$ & $\begin{array}{l}s_{0} s_{1} s_{2} s_{5} s_{3} s_{4} s_{2} s_{3} s_{1} s_{2} s_{5} s_{0} s_{1} \\
s_{5} s_{1} s_{2} s_{3} s_{0} s_{1} s_{2} s_{5} s_{4} s_{3} s_{2} s_{1} s_{0} \\
s_{1} s_{2} s_{5} s_{3} s_{4} s_{2} s_{3} s_{1} s_{2} s_{5} s_{0} s_{1} s_{2} \\
s_{2} s_{5} s_{1} s_{2} s_{3} s_{0} s_{1} s_{2} s_{5} s_{4} s_{3} s_{2} s_{1}\end{array}$ \\
\hline$E_{7}$ & $\bullet \bullet_{0}-\bullet_{1}-\bullet_{2}-\bullet_{3}-\bullet_{4}-\bullet_{6}$ & $\begin{array}{l}s_{0} s_{1} s_{2} s_{3} s_{4} s_{6} s_{5} s_{2} s_{3} s_{4} s_{1} s_{2} s_{3} s_{0} s_{1} \\
s_{3} s_{4} s_{6} s_{1} s_{2} s_{3} s_{0} s_{1} s_{2} s_{5} s_{4} s_{3} s_{2} s_{1} s_{0} \\
s_{1} s_{2} s_{3} s_{4} s_{6} s_{5} s_{2} s_{3} s_{4} s_{1} s_{2} s_{3} s_{0} s_{1} s_{2} \\
s_{2} s_{3} s_{4} s_{6} s_{1} s_{2} s_{3} s_{0} s_{1} s_{2} s_{5} s_{4} s_{3} s_{2} s_{1} \\
s_{5} s_{2} s_{3} s_{4} s_{6} s_{1} s_{2} s_{5} s_{3} s_{4} s_{2} s_{3} s_{0} s_{1} s_{2} s_{5}\end{array}$ \\
\hline
\end{tabular}

Fig. 1: Minimal non-Deodhar patterns

Theorem 2.6 (Deo90), (BW01), (BJ07) Let $W$ be a finite Weyl group, and let $\mathrm{w}=\mathrm{w}_{1} \cdots \mathrm{w}_{k}$ be a reduced expression for some $w \in W$. Then the following are equivalent:

1. The element $w$ is Deodhar.

2. For all $x \in W$, the Kazhdan-Lusztig polynomial $P_{x, w}$ is given by

$$
P_{x, w}(q)=\sum q^{d(\sigma)}
$$

where the sum is over all masks $\sigma$ on $\mathrm{w}$ such that $\mathrm{w}^{\sigma}=x$. 
3. The Kazhdan-Lusztig basis element $C_{w}^{\prime}$ satisfies $C_{w}^{\prime}=C_{\mathrm{w}_{1}}^{\prime} \cdots C_{\mathrm{w}_{k}}^{\prime}$.

4. The Bott-Samelson resolution $f_{\mathrm{w}}: Z \rightarrow X_{w}$ of the corresponding Schubert variety $X_{w}$ is small, so $I H_{*}\left(X_{w}\right) \cong H_{*}(Z)$.

5. $w$ avoids the list of embedded factor patterns given in Figure 1, as well as the 1-line pattern $[\overline{1} 678 \overline{5} 234]$ in type $D$.

The equivalence of (1) through (4) are implicit in Deodhar (Deo90). Lusztig (Lus93) and Fan and Green (FG97) have studied those elements for which (3) holds. These elements are called "tight" in the terminology of those papers. Item (5) is a combinatorial characterization of the Deodhar elements which can also be used to give a polynomial time algorithm for testing whether an element is Deodhar. Billey and Warrington (BW01) first proved (5) for type $A$ and this result has been generalized in (BJ07) for the other finite Weyl groups.

\section{The 0-1 property for $\mu$ coefficients}

Using Deodhar's framework together with combinatorial arguments on heaps, we are able to show that when $w$ is a Deodhar element of a finite Weyl group, the coefficient of $q^{\frac{1}{2}(l(w)-l(x)-1)}$ in the KazhdanLusztig polynomial $P_{x, w}(q)$ is always either 0 or 1 . This notorious coefficient is known as $\mu(x, w)$ in the literature and corresponds to the term of highest possible degree in $P_{x, w}(q)$. The $\mu(x, w)$ values appear in multiplication formulas for the Kazhdan-Lusztig basis elements $\left\{C^{\prime}{ }_{w}\right\}$ of the Hecke algebra and are used in the construction of Kazhdan-Lusztig graphs and cells which in turn are used to construct Hecke algebra representations. This was first done in (KL79) and is described in detail for type $A$ by (BB05). Since the $\mu$ coefficients control the recursive structure of the Kazhdan-Lusztig polynomials, computing $\mu(x, w)$ is not known to be any easier than computing the entire Kazhdan-Lusztig polynomial $P_{x, w}(q)$.

This result is motivated by the following open problem.

Question 3.1 Fix a finite Coxeter group $W$. Is there a simple characterization of the elements $w \in W$ such that $\mu(x, w) \in\{0,1\}$ for all $x \in W$ ?

When all of the elements $w$ in a left cell $\mathcal{C}$ of the finite Weyl group $W$ satisfy $\mu(x, w) \in\{0,1\}$ for all $x \in W$, then Kazhdan-Lusztig's construction of the representation of $W$ associated to $\mathcal{C}$ depends only on the underlying graph structure of the $W$-graph, rather than the edge-labeling of the graph by $\mu$ coefficients. See (KL79) or Chapter 6 of (BB05) for details.

Until fairly recently, it was conjectured that all $\mu(x, w)$ for type $A$ were either 0 or 1 . See (MW03) for some history about this conjecture and an example in $S_{10}$ that shows the conjecture to be false. On the other hand, if $w$ and $x$ are elements of a finite Weyl group then under each of the hypotheses below we have $\mu(x, w) \in\{0,1\}$.

(1) Suppose $w \in S_{n}$, the symmetric group with $n \leq 9$.

(2) Suppose $w$ is a covexillary permutation (equivalently, the 1-line notation for $w$ avoids the permutation pattern [3412]).

(3) Suppose $w$ corresponds to a smooth Schubert variety.

(4) Suppose $w$ and $x$ are fully commutative elements of types $A, D$ or $E$. 
(5) Suppose $w, x \in S_{n}$, the symmetric group, with $a(x)<a(w)$ where $a: S_{n} \rightarrow \mathbb{N}$ is the function defined by Lusztig in (Lus85).

We have (1) from a difficult verification due to (MW03). We have (2) from a formula developed in (LS81) and (Las95) for $P_{x, w}$ using certain edge labeled trees. When the Schubert variety associated to $w$ is smooth, we have by (KL80) that $P_{x, w}(q)=1$ for all $x$, proving (3). In type $A$, we mention that $w$ is $\{[3412],[4231]\}$-avoiding if and only if $w$ is smooth by (LS90; Rya87; Wol89). In (Gra95) (4) was observed for types $A, D$ and $E$. We have (5) due to (Xi05). Note that (4) and (5) require a restriction on both $x$ and $w$.

Our result adds a new set of elements to the list above.

Theorem 3.2 (Jon07b) If $w$ is a Deodhar element of a finite Weyl group $W$ and $x \in W$ then $\mu(x, w) \in$ $\{0,1\}$.

This theorem is proved by carefully examining the heaps of Deodhar elements in type $A$. The result then follows quickly for type $B$ essentially because the unlabeled Coxeter graph of type $B$ is the same as the unlabeled Coxeter graph of type $A$. We can also reduce the type $D$ case to the type $A$ result using techniques which were first developed in (BJ07) to prove the characterization in Theorem 2.6. Finally, we verify the result for the other finite Weyl groups by computer.

Although the first $\mu$ coefficient that is larger than 1 does not appear in type $A$ until rank 9 , there are $\mu$ coefficients as large as 4 in $D_{6}$ and as large as 6 in $E_{6}$. This can be verified using the program Coxeter 1.01 of Fokko du Cloux (dC).

\section{Kazhdan-Lusztig polynomials for maximally-clustered hexagon- avoiding permutations}

In (Los07), Losonczy introduced the maximally clustered elements of simply laced Coxeter groups. We define a set of masks for the maximally-clustered hexagon-avoiding permutations that generalizes Theorem 2.6 in type $A$.

Definition 4.1 (Los07) $A$ braid cluster is an expression of the form

$$
s_{i_{1}} s_{i_{2}} \ldots s_{i_{k}} s_{i_{k+1}} s_{i_{k}} \ldots s_{i_{2}} s_{i_{1}}
$$

where each $s_{i_{p}}$ for $1 \leq p \leq k$ has a unique $s_{i_{q}}$ with $p<q \leq k+1$ such that $\left|i_{p}-i_{q}\right|=1$.

Let $w$ be a permutation and let $N(w)$ denote the number of [321] pattern instances in $w$. We say $w$ is maximally-clustered if there is a reduced expression for $w$ of the form

$$
a_{0} c_{1} a_{1} c_{2} a_{2} \ldots c_{M} a_{M}
$$

where each $a_{i}$ is a reduced expression, each $c_{i}$ is a braid cluster with length $2 n_{i}+1$ and $N(w)=\sum_{i=1}^{M} n_{i}$. Such an expression is called contracted. In particular, $w$ is freely-braided if there is a reduced expression for $w$ with $N(w)$ disjoint short-braids.

Note that this is not the original definition for the maximally clustered elements; however it is equivalent. The remarks in Section 5 of (GL02) show that the number of [321] pattern instances in $w$ equals the number of contractible triples of roots in the inversion set of $w$. Corollary 4.3.3 (ii) and Corollary 4.3.5 
of (Los07) prove that $w$ is a contracted reduced expression for a maximally-clustered element if and only if it has the form given in Definition 4.1. Observe that a maximally-clustered permutation $w$ is fullycommutative if and only if $N(w)=0$ by (BJS93).

In type $A$, there exists a standard form for the braid clusters.

Lemma 4.2 (Jon07a) Suppose $x=s_{i_{1}} s_{i_{2}} \ldots s_{i_{k}} s_{i_{k+1}} s_{i_{k}} \ldots s_{i_{2}} s_{i_{1}}$ is a braid cluster of length $2 k+1$ in type A. Then, $x=s_{m+1} s_{m+2} \ldots s_{m+k} s_{m+k+1} s_{m+k} \ldots s_{m+2} s_{m+1}$ for some $m$.

For our work, we will implicitly assume that any braid cluster has the canonical form of Lemma 4.2. Also, we refer to $s_{m+k} s_{m+k+1} s_{m+k}$ as the central braid of the braid cluster

$$
s_{m+1} s_{m+2} \ldots s_{m+k} s_{m+k+1} s_{m+k} \ldots s_{m+2} s_{m+1} .
$$

Given a contracted expression $w$ for a maximally-clustered hexagon-avoiding permutation, we can identify a set of masks on w that turn out to be bounded and admissible, as described in Definition 2.3. Moreover, this set has a simple non-recursive description.

Definition 4.3 Let $\mathrm{w}$ be a contracted expression for a maximally-clustered hexagon-avoiding permutation, where each braid cluster has the form given in Lemma 4.2. We say that a mask $\sigma$ on $\mathrm{w}$ has a $10 \star$-instance if it has the values

$$
\left(\begin{array}{ccccc}
\cdots & s_{i} & s_{i+1} & s_{i} & \ldots \\
* & 1 & 0 & * & *
\end{array}\right)
$$

on any central braid instance $s_{i} s_{i+1} s_{i}$ of any braid cluster in $\mathrm{w}$, where $*$ denotes an arbitrary mask value. If $\sigma$ never has the values 1 and 0 (respectively) on the first two entries in any central braid of $\mathrm{w}$, then we say that $\sigma$ is a 10 *-avoiding mask for $\mathrm{w}$.

Theorem 4.4 (Jon07a) Let $\mathrm{w}$ be a contracted expression for a maximally-clustered hexagon-avoiding permutation in $S_{n}$, and let $\mathcal{E}_{\mathrm{w}}$ be the set of 10 *-avoiding masks on $\mathrm{w}$. Then for any $x \in S_{n}$,

$$
P_{x, w}(q)=P_{x}\left(\mathcal{E}_{\mathrm{w}}\right)=\sum_{\substack{\sigma \in \mathcal{E}_{\mathrm{w}} \\ \mathrm{w}^{\sigma}=x}} q^{d(\sigma)} .
$$

This result follows from Theorem 2.4 once it is shown that $\mathcal{E}_{\mathrm{w}}$ is bounded and admissible. That $\mathcal{E}_{\mathrm{w}}$ is bounded follows from an inductive argument which involves the heap associated to w. To prove that $\mathcal{E}_{\mathrm{w}}$ is admissible, we must show that it satisfies the three properties in Definition 2.3. The first property is clear since the mask $\sigma=(1,1, \ldots, 1)$ avoids $10 *$, and the second property holds because avoiding 10 * imposes no restrictions on the last entry of a mask.

In order to show that $h\left(\mathcal{E}_{\mathrm{w}}\right)$ is invariant under the Hecke algebra involution, we use that

$$
h\left(\mathcal{E}_{\mathrm{w}}\right)=\overline{h\left(\mathcal{E}_{\mathrm{w}}\right)} \text { if and only if } h\left(\mathcal{E}_{\mathrm{w}}^{c}\right)=\overline{h\left(\mathcal{E}_{\mathrm{w}}^{c}\right)}
$$

where $\mathcal{E}_{\mathrm{w}}^{c}$ denotes the set complement $\mathcal{S} \backslash \mathcal{E}_{\mathrm{w}}$. We decompose this complement $\mathcal{E}_{\mathrm{w}}^{c}$ so that the invariance under the involution follows from an induction on the number of short-braids in the contracted expression w. 


\section{Acknowledgements}

We wish to thank Sara Billey, Francesco Brenti, Richard Green, Jozsef Losonczy, Monty McGovern, Yuval Roichman, Monica Vazirani and Greg Warrington for many useful conversations and suggestions while this work was being carried out.

\section{References}

[BB81] A. Beilinson and J. Bernstein. Localization of $\mathfrak{g}$-modules. C. R. Acad.Sci. Paris Ser. I Math, 292:15-18, 1981.

[BB05] Anders Björner and Francesco Brenti. Combinatorics of Coxeter groups, volume 231 of Graduate Texts in Mathematics. Springer, New York, 2005.

[BJ07] Sara C. Billey and Brant C. Jones. Embedded factor patterns for Deodhar elements in Kazhdan-Lusztig theory. Ann. Combin., 11(3/4):285-333, 2007. arXiv:math/0612043 [math. CO].

[BJS93] Sara Billey, William Jockusch, and Richard P. Stanley. Some combinatorial properties of Schubert polynomials. J. Algebraic Combin., 2(4):345-374, 1993.

[BK81] J.-L. Brylinski and M. Kashiwara. Kazhdan-Lusztig conjectures and holonomic systems. Invent. Math., 64:387-410, 1981.

[BW01] Sara Billey and Gregory S. Warrington. Kazhdan-Lusztig polynomials for 321-hexagonavoiding permutations. J. Algebraic Combin., 13(2):111-136, 2001.

[dC] Fokko du Cloux. http://igd.univ-lyon1.fr/ ducloux/coxeter/coxeter1/ coxeter_e.html.

[Deo90] Vinay V. Deodhar. A combinatorial setting for questions in Kazhdan-Lusztig theory. Geom. Dedicata, 36(1):95-119, 1990.

[Fan98] C. K. Fan. Schubert varieties and short braidedness. Transform. Groups, 3(1):51-56, 1998.

[FG97] C. K. Fan and R. M. Green. Monomials and Temperley-Lieb algebras. J. Algebra, 190(2):498517, 1997.

[GL02] R. M. Green and J. Losonczy. Freely braided elements of Coxeter groups. Ann. Comb., 6(34):337-348, 2002.

[Gra95] John J. Graham. Modular Representations of Hecke Algebras and Related Algebras. PhD thesis, University of Sydney, 1995.

[Gre07] Richard M. Green. Generalized Jones traces and Kazhdan-Lusztig bases. Preprint; to appear in J. Pure Appl. Algebra, 2007.

[Jon07a] Brant C. Jones. Kazhdan-Lusztig polynomials for maximally-clustered hexagon-avoiding permutations. arXiv:0704.3067 [math.CO], 2007. 
[Jon07b] Brant C. Jones. Leading coefficients of Kazhdan-Lusztig polynomials for Deodhar elements. arXiv:0711.1391 [math.CO], to appear in J. Algebraic Combin., 2007.

[KL79] David Kazhdan and George Lusztig. Representations of Coxeter groups and Hecke algebras. Invent. Math., 53(2):165-184, 1979.

[KL80] D. Kazhdan and G. Lusztig. Schubert varieties and Poincaré duality. Proc. Symp. Pure. Math., A.M.S., 36:185-203, 1980.

[Los07] J. Losonczy. Maximally clustered elements and Schubert varieties. Ann. Comb., 11:195-212, 2007.

[Las95] Alain Lascoux. Polynômes de Kazhdan-Lusztig pour les variétés de Schubert vexillaires. (French) [Kazhdan-Lusztig polynomials for vexillary Schubert varieties]. C. R. Acad. Sci. Paris Sér. I Math., 321(6):667-670, 1995.

[LS81] Alain Lascoux and Marcel-Paul Schützenberger. Polynômes de Kazhdan \& Lusztig pour les grassmanniennes. In Young tableaux and Schur functors in algebra and geometry (Toruń, 1980), volume 87 of Astérisque, pages 249-266. Soc. Math. France, Paris, 1981.

[LS90] V. Lakshmibai and B. Sandhya. Criterion for smoothness of Schubert varieties in $\mathrm{Sl}(n) / B$. Proc. Indian Acad. Sci. Math. Sci., 100(1):45-52, 1990.

[Lus85] George Lusztig. Cells in affine Weyl groups. In Algebraic groups and related topics (Kyoto/Nagoya, 1983), volume 6 of Adv. Stud. Pure Math., pages 255-287. North-Holland, Amsterdam, 1985.

[Lus93] G. Lusztig. Tight monomials in quantized enveloping algebras. In Quantum deformations of algebras and their representations (Ramat-Gan, 1991/1992; Rehovot, 1991/1992), volume 7 of Israel Math. Conf. Proc., pages 117-132. Bar-Ilan Univ., Ramat Gan, 1993.

[MW03] Timothy J. McLarnan and Gregory S. Warrington. Counterexamples to the 0-1 conjecture. Represent. Theory, 7:181-195 (electronic), 2003.

[Rya87] Kevin M. Ryan. On Schubert varieties in the flag manifold of Sl(n, C). Math. Ann., 276(2):205224, 1987.

[SS85] Rodica Simion and Frank W. Schmidt. Restricted permutations. European J. Combin., 6(4):383-406, 1985.

[Ste96] John R. Stembridge. On the fully commutative elements of Coxeter groups. J. Algebraic Combin., 5(4):353-385, 1996.

[Tit69] Jacques Tits. Le problème des mots dans les groupes de Coxeter. In Symposia Mathematica (INDAM, Rome, 1967/68), Vol. 1, pages 175-185. Academic Press, London, 1969.

[Wo189] James S. Wolper. A combinatorial approach to the singularities of Schubert varieties. Adv. Math., 76(2):184-193, 1989.

[Xi05] Nanhua Xi. The leading coefficient of certain Kazhdan-Lusztig polynomials of the permutation group $S_{n}$. J. Algebra, 285(1):136-145, 2005. 